\title{
The safety and well-being of everyday heroes
}

\section{Norman E. Taylor*}

It is an unfortunate reality that for those professionals whose careers are dedicated to ensuring the safety, health, and wellbeing of others, every day at work carries with it a unique set of risk factors to themselves. Our Journal is very proud to dedicate this issue to these everyday heroes.

In February 2017, over 200 delegates came together in Gatineau, QC to participate in the third joint conference on mental health issues co-presented by the Canadian Association of Chiefs of Police (CACP) and the Mental Health Commission of Canada (MHCC). Though the conference was titled as the "Mental Health of Police Personnel", the delegates recognized and the agenda reflected that police are certainly not the only first responders or human service providers to be affected by routine stressors and exceptional traumas in service of others. Several of the conference sessions cut across the full spectrum of those so affected, and also included issues relating to responders' families, and considerations for responders who are among the many victims of mass casualty events. In this issue, we feature our first Record of Proceedings from this international event, effectively captured and summarized from the two-day conference by Ahlgren.

As well, from among several thought-provoking sessions that shaped that event, we are pleased to feature an exemplary narrative by Klose, Mooney \& McLeod, describing one police agency's innovative approach to supporting members through their return-to-work challenges brought about by traumatic events. And on a related track, Anderson, Vaughan \& Mills deliver an original research paper examining the effectiveness and potential of online training for building resilience in paramedic students.
Like all our issues of the Journal, this one also continues to broaden our discussions and deepen our understanding around the needs and solutions for vulnerable individuals and communities in general. We hope our readers will find value in each of these articles, as well.

But, I would ask that, as you read through the features in this issue that address persons in our communities facing the challenges of addictions and mental health issues, and our collective measurement of promising CSWB practices, collaborative and supportive services, and the better outcomes that result ... please take a moment to reflect on the other side of that equation.

\section{CONFLICT OF INTEREST DISCLOSURE}

The author has no known conflicts of interest.

\section{AUTHOR AFFILIATIONS}

*Editor-in-Chief, Journal of Community Safety and Well Being.

\section{REFERENCES}

Ahlgren, A. (2017). The mental health of police personnel: what we know \& what we need to know and do (CACP-MHCC Conference 13-15 February 2017). Journal of Community Safety and Well-Being, 2(2).

Anderson, G. S., Vaughan, A. D., Mills, S. (2017). Building personal resilience in paramedic students. Journal of Community Safety and Well-Being, 2(2).

Klose, G., Mooney, C., Mcleod, D. (2017). Re-integration: a new standard in 1st responder peer support. Journal of Community Safety and WellBeing, 2(2). 